\title{
Model of chromosome associations in Mus domesticus spermatocytes
}

\author{
Soledad Berríos ${ }^{1}$, Marcia Manterola' ${ }^{1}$ Zulita Prieto², Julio López-Fenner ${ }^{4}$, Jesús Page ${ }^{3}$ \\ and Raúl Fernández-Donoso ${ }^{*}$
}

${ }^{1}$ Programa Genética Humana, ICBM, Facultad de Medicina, Universidad de Chile.
2 Departamento de Biología, Universidad Nacional de Trujillo, Perú.
${ }_{3}^{3}$ Departamento de Biología Celular, Universidad Autónoma de Madrid, España.
${ }^{4}$ Departamento de Ingeniería Matemática, Universidad de La Frontera, Temuco.

\begin{abstract}
Understanding the spatial organization of the chromosomes in meiotic nuclei is crucial to our knowledge of the genome's functional regulation, stability and evolution.

This study examined the nuclear architecture of Mus domesticus $2 \mathrm{n}=40$ pachytene spermatocytes, analyzing the associations among autosomal bivalents via their Centromere Telomere Complexes (CTC).

The study developed a nuclear model in which each CTC was represented as a 3D computer object. The probability of a given combination of associations among CTC was estimated by simulating a random distribution of 19 indistinguishable CTC over $n$ indistinguishable "cells" on the nuclear envelope. The estimated association frequencies resulting from this numerical approach were similar to those obtained by quantifying actual associations in pachytene spermatocyte spreads. The nuclear localization and associations of CTC through the meiotic prophase in well-preserved nuclei were also analyzed. We concluded that throughout the meiotic prophase: 1) the CTC of autosomal bivalents are not randomly distributed in the nuclear space; 2) the CTC associate amongst themselves, probably at random, over a small surface of the nuclear envelope, at the beginning of the meiotic prophase; 3 ) the initial aggregation of centromere regions occurring in lepto-zygotene likely resolves into several smaller aggregates according to patterns of preferential partitioning; 4) these smaller aggregates spread over the inner face of the nuclear envelope, remaining stable until advanced stages of the meiotic prophase or even until the first meiotic division.
\end{abstract}

Key terms: bivalent associations, nuclear model, mouse spermatocytes, heterochromatin distribution.

\section{INTRODUCTION}

Associations among chromosomes contribute significantly to the organization of the nucleus, and their occurrence and maintenance depend on the morphological characteristics of the chromosomes, the functional properties of the chromosome territories, and the type of cell (Nagele et al., 1995; Dernburg et al., 1996; Sadoni et al., 1999; Cerda et al., 1999; Alcobia et al., 2000; Mayer et al., 2005). In situ hybridization, immunocytochemistry, and computational analysis of 3D structures have allowed for major progress in the study of interphasic nuclear organization and the associations or interactions among chromosome territories (Manuelidis 1985; Manuelidis and Borden, 1988; Haaf and Schmid, 1991; Sadoni et al., 1999; Beil et al., 2005; de Wit and van Steensel, 2009). Although we still lack a generalized model to explain chromosome arrangement in the somatic cell nucleus, there is no doubt that interphase chromosome topology is important for the complex regulation of the many varied and interrelated nuclear processes (Haaf and Schmid M., 1991; Cremer et al., 2004; Cremer et al., 2006; Takizawa et al., 2008; Misteli, 2008; Göndor and Ohlsson, 2009).

In meiotic nuclei, specifically during the first prophase, the chromatin profoundly reorganizes its distribution in the nuclei of somatic cells. The chromatin of each chromosome, after replication, binds to axial elements that in turn bind to the nuclear envelope. Then the homologous chromosome pairs synapse, via the formation of the synaptonemal complex (SC), becoming bivalents. The SC is a proteinaceous structure assembled between the two homologues that consists of two lateral elements (LEs), one per homologue, bound in a zipper-like manner, by the central element (CE) (Heyting, 1996; von Wettstein et al., 1984; Yang and Wang, 2009). Two proteins, SYCP2 and SYCP3, are the main components of the LE, while SYCP1 is the main component of the CE (Lammers et al., 1994; Offenberg et al., 1998).

Chromosome pairing and synapsis are the main features of meiosis; however, associations between bivalents are also a normal constituent of meiotic nuclear organization. Preferential associations among bivalents at the nucleolar, centromeric, and/or heterochromatic territories have been described in the prophase meiotic nuclei of spermatocytes and oocytes of various species. The movement of two or more bivalents towards a large nucleolus, which is the product of heterologous NOR genetic activity, has been observed, as has the aggregation of various bivalents via the pericentromeric heterochromatin. The heterochromatin forms one or more condensed chromocenters that appear as clusters of chromatin that are denser than the other nuclear components (Stahl et al. 1984; Zuccoti et al., 1995; Berrios and Fernández-Donoso, 1990; Berrios et al., 2004).

In Mus domesticus $2 \mathrm{n}=40$ the pericentromeric heterochromatin appears as large blocks in the proximal terminals of the chromosomes and extends towards the centromere and proximal telomere (Pardue and Gall., 1970; Redi et al., 2001). Molecular exploration of the centromeric regions of Mus has revealed that there are two types of

* Corresponding author: R Fernández-Donoso (e-mail: rfernand@med.uchile.cl)

Received: October 29, 2009. In revised form: May 13, 2009. Accepted: June 8, 2010. 
subterritories, the major and minor satellites, with distinct repetitive DNA sequences and different functions in the nucleus (Garagna et al., 2002). The minor satellite is associated with the functioning of the centromere itself, while the major satellite is part of the heterochromatin responsible for the aggregation of various cluster-forming chromosomes (Joseph, et al., 1989; Garagna et al., 2002; Guenatri et al., 2004). It has been demonstrated that the pericentromeric heterochromatin and chromocenters in the spermatocytes of various species of mammals do not aggregate randomly, but rather in a species-specific pattern (Solari and Tres 1970; Berrios et al., 1999). It has been also described that throughout mouse pachytene, the kinetochores are arranged non-randomly into several clusters and are distributed around the periphery of the nucleus (Brinkley et al., 1986, Berrios et al., 1999). It has been proposed that the distribution of heterochromatin and centromeres in the nuclear periphery of Mus domesticus pachytene spermatocytes is determined by the end position of the heterochromatin in the bivalents and by the organization of the meiotic prophase, during which the telomeres of the bivalents are bound to the nuclear envelope (Fernández-Donoso, 1982; Berrios et al., 1999).

In Mus spermatocytes, there are 19 autosomal bivalents, all with similar terminal centromeres and all carriers of abundant heterochromatin. The bivalents form associations among themselves via their heterochromatic regions, giving rise to a variable number of chromocenters and single bivalents. The phenomenon of physical association among heterologous territories is important in determining the meiotic nuclear architecture and consequently its genomic functionality. This is an area that has been under-studied. Characterizing and quantifying observed associations can lead to significant conclusions only if there are reference values with which to compare them. To this end, the present study sought to design a computational model of the nuclear architecture based on 3D imagery of the nucleus and the participating bivalents, and a probabilistic nuclear model that predicts which theoretical and probable combinations of associations among heterochromatic regions are predetermined and which are random. Furthermore, the study quantifies the observed number and characteristics of the associations among the bivalents in $400 \mathrm{Mus}$ domesticus $2 n=40$ spermatocytes. The resulting observations are then compared with the model simulations of the nuclear architecture and the predictions of the probabilistic model. In this context, the authors discuss the origin of associations among the proximal heterochromatic territories during the bouquet stage of early meiotic prophase, the formation of various chromocenters as prophase progresses, and the resolution or persistence of this suprachromosomic organization through meiotic division I.

\section{METHODS}

Definition of CTC and simulation of associations between CTC on the nuclear envelope

In the terminal centromere of the Mus domesticus bivalents $2 n=40$ there is a complex chromosomal entity whose basic organization is common to all bivalents. This entity is formed by the proximal telomere (Tp), the centromere, and the pericentromeric heterochromatin, including the segment of the synaptonemal complex (SC) found in this region. Given the regularity with which the centromeric region is organized in all Mus autosomal bivalents, we computationally designed a 3D object to represent the size, form, and constituent elements of this entity. We named this object the Centromere Telomere Complex (CTC) in allusion to its constituent elements and their structural unity (Fig. 1a). An approximately $1 \mu \mathrm{m}^{2}$ portion of the CTC surface adheres to the internal face of the nuclear envelope (Fig. 1b). We simulated the possible distributions of the CTC over the nuclear envelope, including variations in the ways in which the 19 CTC may associate (Figs. 1c, 1d and 2). For example, one CTC may be surrounded by another 6 CTC, forming an association of 7 , or the same 7 CTC may be bound to one another on two sides only, resulting in a closed or open linear association. In either case, the remaining 12 CTC (of the 19 total) may be present in various associations or remain single. These simulations demonstrate that the potential combinations of associations and non-associations of the 19 CTC in each spermatocyte nucleus are numerous (Fig. 2). We developed a list of all possible combinations of single or associated CTC per spermatocyte and estimated the probability of the occurrence of each, with the goal of establishing a theoretical reference value with which we could contrast the observed frequencies of associations in the spermatocytes.

Nuclear model and expected probabilities of associations among CTC

In this model of nuclear architecture, we obtained numerical estimates of the probabilities of associations among CTC. The 19 mouse CTC were treated as indistinguishable from one another. The union of each CTC with the internal face of the nuclear envelope occupies an area of $1 \mu \mathrm{m}^{2}$, which was represented by a hexagon, referred to as a cell. We considered two CTC to be associated if they occupied contiguous cells. In a Mus spermatocyte, during the meiotic prophase nucleus measures approximately $10 \mu \mathrm{m}-15 \mu \mathrm{m}$ in diameter. For example, in a nucleus of $14 \mu \mathrm{m}$ diameter, the surface of the nuclear envelope is approximately $620 \mu \mathrm{m}^{2}$, containing 620 cells. We estimated the probability of associations among 19 identical CTC distributed randomly over various areas of the nuclear envelope, with the corresponding conjunctions of cells also deemed indistinguishable from one another. In these simulations we considered the total surface area of the nuclear envelope (620 cells); the area corresponding to a nuclear hemisphere (or 310 cells); or an area of approximately $8-10 \%$ of the total surface area of the prophase nuclear envelope (or 51 contiguous cells). This last area represents the minimum number of cells capable of containing the 19 CTC, 19 distal telomeres, and telomeres of the $X Y$ bivalent (Fig. 2a). This area is also representative of the bouquet stage of early prophase, in which all telomeres are clustered on a reduced area of the nuclear envelope. Of all the simulations performed, the distribution over 51 cells was most consistent with the observed results. For the simulations, the XY bivalent was not treated as a potential CTC association component, as it is a distinguishable element with a partial synapse among its chromosomes. We calculated the possible CTC combinations according to the algorithm developed by 


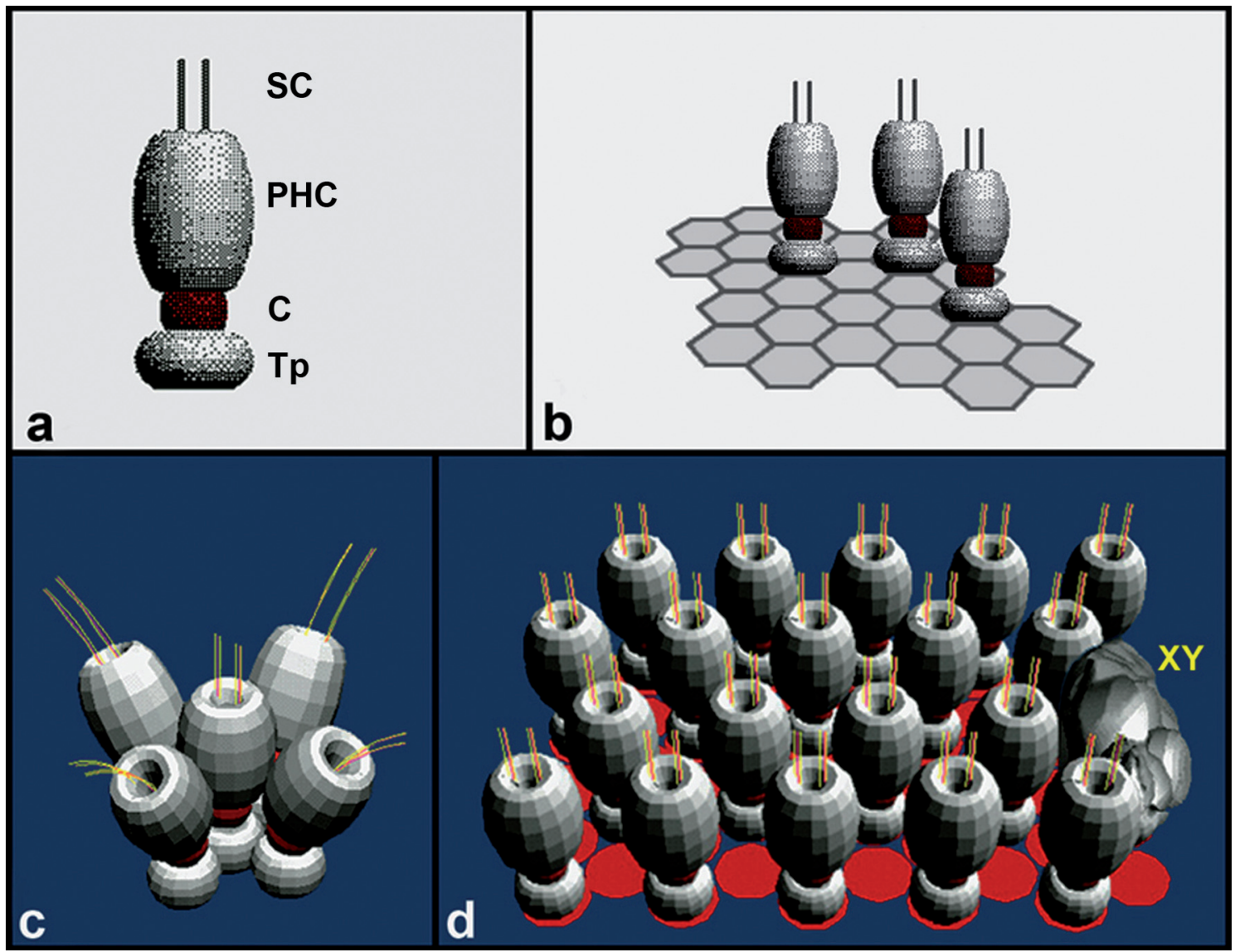

Figure 1: Computational representation of a CTC and its position over the cells

a) 3D representation of the shape and dimensions of a CTC. Each CTC consists of a proximal telomere (TP), centromere (C), and pericentromeric heterochromatin ( $\mathrm{PCH})$, including the segment of the Synaptonemal Complex (SC) found in that region; b) Three CTC positioned over the nuclear envelope, each connected to a cell via its proximal telomere (Tp); c) Association of 5 CTC, each CTC equivalent in size and organization, treated as equivalent; d) Distribution of 19 non-associated, equivalent CTC and the XY bivalent over 51 contiguous cells (Distribution equivalent to that simulated in Figure 2a).

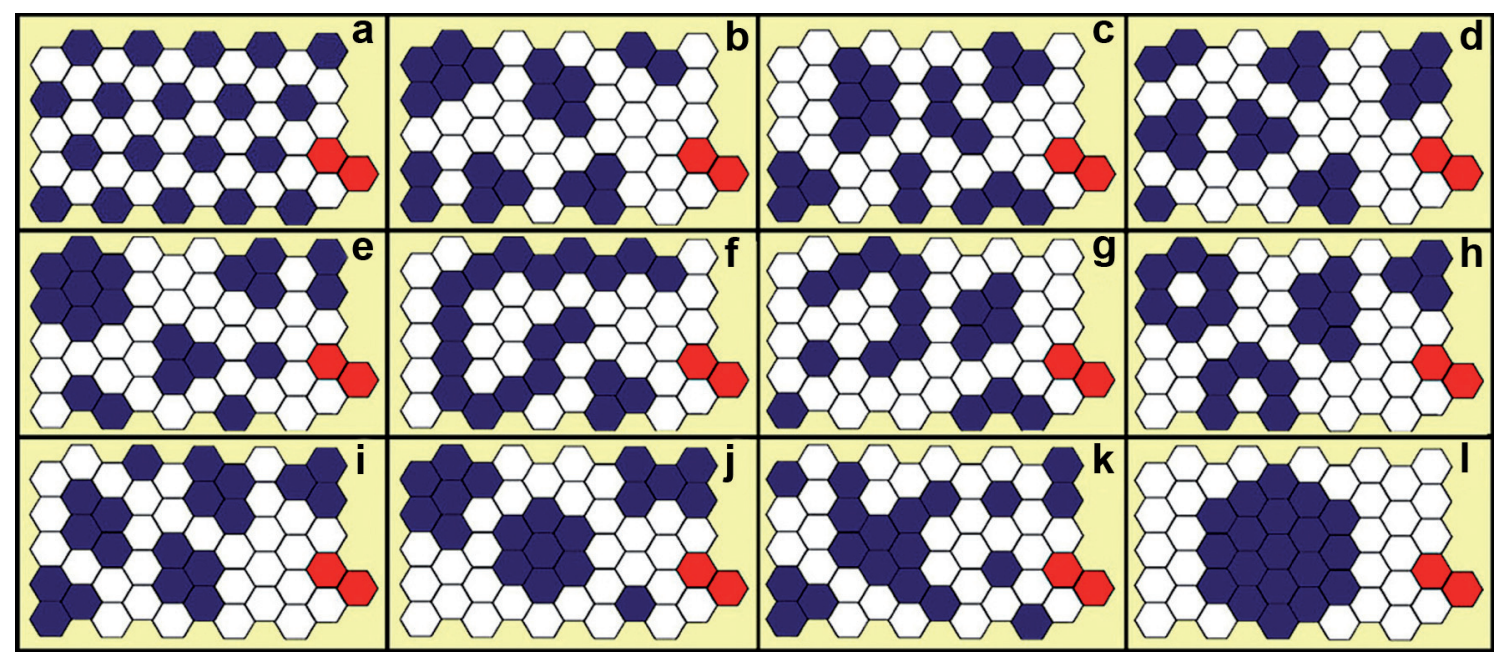

Figure 2: Possible distributions of the 19 CTC over 51 contiguous cells representing a portion of the nuclear envelope surface Each hexagon or cell corresponds to a surface area of $1 \mathrm{um}^{2}$, which may be: occupied by a CTC (blue), occupied by a distal telomere (Tq), unoccupied (white), or occupied by the XY bivalent (red). The figure shows 12 combinations of the possible 490 (Table 1 ). a) the CTC are not associated; b) there is one association of 5 CTC, another of 4, two of 3, and two of 2, or the combination 5-4-3-3-2-2; c) $5-3-3-3-3-2$; d) 4-3-3-3-3-2-1; e) 7,-3-3-2-2-1-1; f) 16-3; g) 7-4-3-2-1-1; h) 6-5-5-3; i) 4-4-4-3-3-1; j) 7-6-5-1; k) 10-3-2-1-1-1-1 and I) all 19 CTC are associated. The classes to which the combinations belong are the following: a) Class 1 ; b) Class 5; c) Class 5; d) Class 4; e) Class 7; f) Class 16; g) Class 7; h) Class 6; i) Class 4; j) Class 7; k) Class 10; l) Class 19. 
López-Fenner and Fernández-Donoso (1991), resulting in 490 equiprobable numerical combinations of associated and nonassociated CTC (Table 1). We grouped these combinations into 19 classes defined according to the largest grouping of CTC within the combination. Each class includes all combinations containing this value. Figure 2 illustrates 12 of 490 possible combinations: a) the CTC are all single; b) there is one association of 5 CTC, another of 4 , two of 3 , and two of 2 , or the combination 5-4-3-3-2-2; c) 5-3-3-3-3-2; d) 4-3-3-3-3$2-1$; e) $7,-3-3-2-2-1-1$; f) $16-3$; in g) $7-4-3-2-1-1$; h) $6-5-5-3$; i) $4-$ 4-4-3-3-1; in j) 7-6-5-1; in k) 10-3-2-1-1-1-1; and 1) all 19 CTC are associated. The classes to which these combinations belong are the following: a) Class 1 ; b) Class 5; c) Class 5; d) Class 4; e) Class 7; f) Class 16; g) Class 7; h) Class 6; i) Class 4 ; j) Class 7; k) Class 10; 1) Class 19. For example, Figures 2e, $\mathrm{g}$, and $\mathrm{j}$ all represent Class 7 combinations, with varied forms of aggregation among the CTC, that is, clusters, open or closed lines. These variations within a given class are not differentiated in the simulation.

We estimated the number of combinations in each of the 19 classes, and assuming that all combinations were equiprobable, calculated the frequency of each class (Table 1). The expected frequencies had an approximately normal distribution, with a leftward deviation (Fig 5, black bars). The highest-frequency combinations were about $10-14 \%$, for Classes 4 and 8. Classes 1 and 16-19 had frequencies below $1 \%$ (Table 1).

Table 1

Number of combinations of single and associated CTC per class and expected probability of each class.

\begin{tabular}{|c|c|c|}
\hline Classes & $\begin{array}{c}\text { Number of CTC } \\
\text { combinations per class }\end{array}$ & $\begin{array}{c}\text { Expected probability } \\
\text { of each class }\end{array}$ \\
\hline 1 & 1 & 0.204 \\
\hline 2 & 9 & 1.837 \\
\hline 3 & 30 & 6.122 \\
\hline 4 & 54 & 11.020 \\
\hline 5 & 70 & 14.286 \\
\hline 6 & 71 & 14.490 \\
\hline 7 & 65 & 13.265 \\
\hline 8 & 52 & 10.612 \\
\hline 9 & 41 & 8.367 \\
\hline 10 & 30 & 6.122 \\
\hline 11 & 22 & 4.490 \\
\hline 12 & 15 & 3.061 \\
\hline 13 & 11 & 2.245 \\
\hline 14 & 7 & 1.429 \\
\hline 15 & 5 & 1.020 \\
\hline 16 & 3 & 0.612 \\
\hline 17 & 2 & 0.408 \\
\hline 18 & 1 & 0.204 \\
\hline 19 & 1 & 0.204 \\
\hline Total 19 & 490 & 100.00 \\
\hline
\end{tabular}

Spermatocyte nuclear spreads and quantification of single and associated CTC

We analyzed spermatocytes from two male three-month-old Mus domesticus $2 \mathrm{n}=40 \mathrm{C} 3 \mathrm{H}$ mice. Mice were maintained at $22^{\circ} \mathrm{C}$ with a light/dark cycle of $12 / 12$ hours and fed ad libitum. Procedures involving the use of the mice were approved by the animal ethics committee of the Faculty of Medicine, Universidad de Chile.

Spermatocyte spreads were obtained following the procedure described by Peters et al. (1997). Briefly, a testicular cell suspension in $100 \mathrm{mM}$ sucrose was spread onto a slide dipped in 1\% paraformaldehyde in distilled water containing $0.15 \%$ Triton X-100 then left to dry for two hours in a moist chamber. They were subsequently washed with $0.08 \%$ Photoflo (Kodak), air-dried and rehydrated in PBS. The slides were incubated for 24 hours at $4^{\circ} \mathrm{C}$ in a moist chamber with the primary antibodies: mouse anti-SYCP3 1:100 (Santa Cruz, 74569) and rabbit anti-H3K9me3 1:200 (Abcam ab8580). After rinsing in PBS, the slides were incubated with appropriate secondary antibodies diluted 1:100 in PBS: FITC-conjugated donkey anti-mouse IgG, Texas Red-conjugated donkey anti-rabbit IgG (Jackson). Slides were then stained with $1 \mu \mathrm{g} / \mathrm{ml}$ DAPI (4,6-diamidino-2phenylindole). After a final rinse in PBS, the slides were mounted with Vectashield Vector laboratories). Observations were made using a Nikon (Tokyo, Japan) Optiphot or Olympus BX61 microscope equipped with epifluorescence optics, and the images were photographed on a DS camera control unit DS-L1 Nikon or captured with an Olympus DP70 digital camera. All images were processed with Adobe Photoshop CS software.

We quantified the bivalent associations in 400 pachytene spermatocyte spreads treated by immunocytochemical techniques, 130 from one mouse and 270 from another. In each nuclear spread, we verified the presence of the 19 SC corresponding to the autosomal bivalents and then recorded whether they were linked to one another at the heterochromatic region. We determined the combinations of associations that the 19 CTC presented in each of the nuclear spreads.

EM spermatocyte microspreads

Spermatocyte microspreads were obtained for electron microscopy (EM) according to the method described by Solari (1982). Briefly, cells were dropped into $0.5 \% \mathrm{NaCl}$. Microspreads were collected on a slide covered with $0.55 \%$ polystyrene in chloroform. They were then fixed in $4 \%$ paraformaldehyde, $0.03 \%$ SDS in sodium borate buffer and contrasted in $1 \%$ ethanolic phosphotungstic acid. The grid film was retrieved using parafilm. Selected grids were observed and photographed using a Zeiss electron microscope EM-109.

Spermatocyte squashes with 3-D preserved nuclei

Spermatocyte squashes that preserved the nuclear volume were obtained following the procedure described by Page et al (1998). Testes were removed and fixed in $2 \%$ formaldehyde in PBS containing $0.05 \%$ Triton X-100. Pieces of tubules were placed in a drop of fixing solution on a 
slide. They were gently minced with tweezers, and then a coverslip was added. The cells were squashed by exerting pressure on the coverslip. The slides were frozen in liquid nitrogen and coverslips were then removed. The slides were incubated for 45 minutes at $37^{\circ} \mathrm{C}$ in a moist chamber with the primary antibodies: rabbit anti-SYCP3 1:100 (Abcam, ab15093) and mouse anti-CENPA 1:200 (Abcam, ab13939). The slides were then incubated for $30 \mathrm{~min}$ at room temperature with the secondary antibodies: FITCconjugated goat anti-mouse IgG (1:50)(Sigma), or Texas redconjugated goat anti rabbit IgG (1:200)(Jackson). Slides were counterstained with $1 \mu \mathrm{g} / \mathrm{ml}$ DAPI (4,6-diamidino-2phenylindole). Finally, slides were rinsed in PBS and mounted in Vectashield (Vector). Immunolabeled spermatocytes were observed in an Olympus BX61 microscope equipped with a motorized Z-axis, epifluorescence and an Olympus DP70 digital camera. A collection of optical sections was captured using the analiSYS software (Soft Imaging System, Olympus). Images were subsequently analyzed and processed using the public domain software ImageJ (National Institutes of Health, United States;http://rsb.info.nih.gov/ij).

\section{Spermatocyte spreads with $C B G$ banding}

Nuclear spermatocyte spreads from five additional male mice were fixed in methanol acetic acid and treated to obtain CBG banding. The preparations were preserved for at least 3 days at $-18^{\circ} \mathrm{C}$. They were then dropped into $0.2 \mathrm{~N} \mathrm{HCl}$ for 30 minutes at room temperature. They were washed in distilled water and air-dried at room temperature. They were dropped in saturated $\mathrm{Ba}(\mathrm{OH})_{2}$ solution for 5 to 10 minutes at room temperature, washed in distilled water, and incubated in $2 \times S S C$ at $60^{\circ} \mathrm{C}$ for 1 hour. They were washed with distilled water and air-dried. They were stained with 7\% Giemsa solution (Merck) in Sörensen's buffer, pH7, for 50 minutes. We quantified the bivalent associations in 665 pachytene spermatocyte CBG-spreads. In each nuclear spread, we studied the combinations of associations of the 19 autosomal bivalents (not shown). The chromosomal associations in the meiotic first division were also studied.

Observations were made using a Nikon (Tokyo, Japan) Optiphot microscope and the images were photographed on a DS camera control unit DS-L1 Nikon.

\section{RESULTS}

\section{Ultrastructure of single or associated CTC}

In an EM microspread of Mus bivalents, a mass of chromatin with a higher electronic density than the rest of the chromatin was observed in the proximal end of each of the autosomal bivalents. This mass corresponds to the pericentromeric heterochromatin (Fig. 3). This mass of chromatin occupies the entire centromeric and proximal telomeric region, expanding until it reaches a volume of approximately $1 \mu \mathrm{m}^{3}$ (Fig. 3). This chromosomal region is represented as a $3 \mathrm{D}$ computer object that we call the Centromere Telomere Complex (CTC) (Fig. 1a). We found single bivalents (Figs. $3 a$ and $b$ ) and associations among bivalents in the microspreads (Figs. 3c-e). Associations of bivalents were always through the CTC via the proximal terminals. Within the associations, the volume of aggregated heterochromatin was proportional to the number of associated bivalents, which were individualizable by their respective synaptonemal complexes (SC) (Fig. 3c-e).

\section{Number of single or associated CTC}

In each nuclear spread of pachytene spermatocytes, we found that the chromosomal axes were totally synapsed, forming 19 SC corresponding to the autosomal bivalents. The sex chromosomes $X$ and $Y$ presented both chromosomal axes, only linked via a partial synapse (Fig 4a-f). In the proximal end of each autosomal bivalent, we observed the heterochromatin by detecting H3K9me3 (Fig. 4). The structure and conditions of the bivalents, single or associated, observed in the spreads was consistent with those from previous electronic microspreads (Figs. 3 and 4).

The number of single or associated bivalents associated with each spermatocyte was estimated according to the volume of the masses of heterochromatin and the number of SC found within. The spermatocytes were divided into classes according to the combinations of associations presented by the heterochromatic masses (or CTC) (see

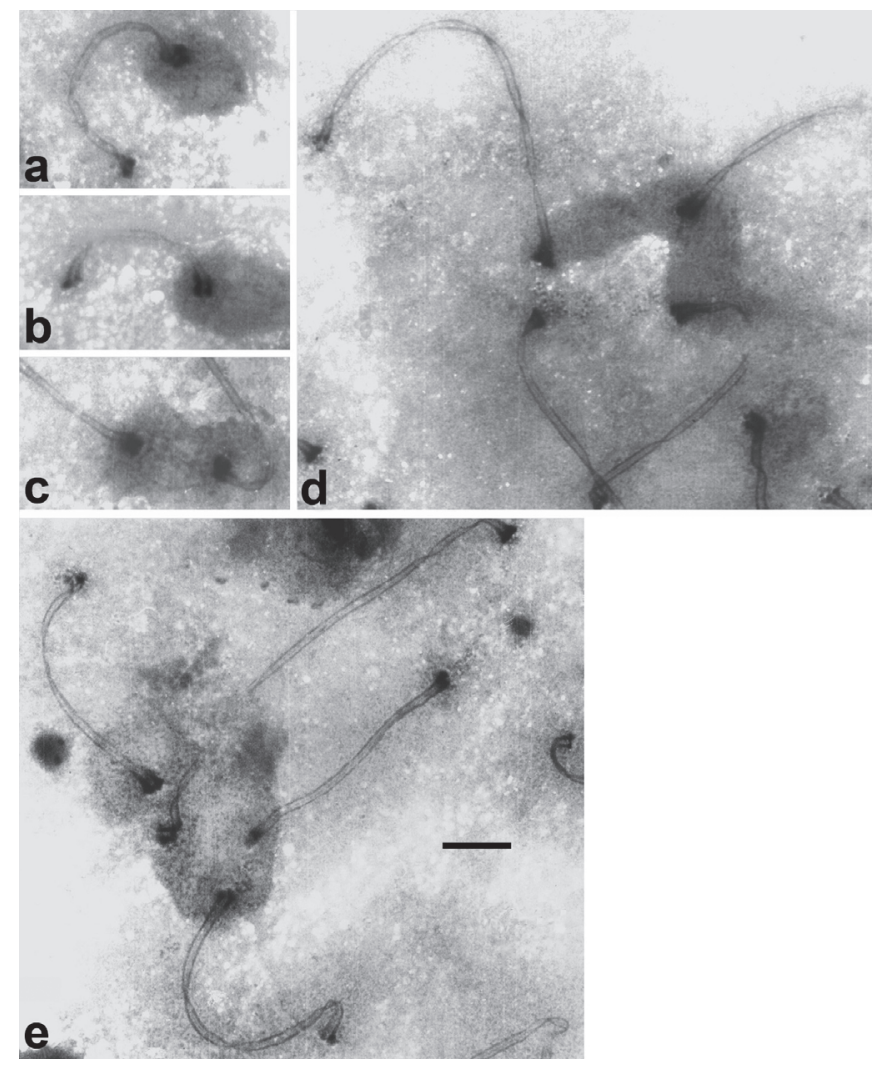

Figure 3: Single and associated CTC in EM microspreads of Mus domesticus $\mathbf{2 n = 4 0}$ spermatocytes

The figure shows a mass of heterochromatin in the CTC of all the bivalents, and the associations among bivalents at the heterochromatin. a) and b) non-associated bivalents; c) association of two bivalents; d) association of three bivalents; e) association of four bivalents. As shown, the size of the pericentromeric heterochromatin mass is proportional to the number of bivalents included in each association. Barr $1 \mathrm{um}$. 
Materials and Methods). Table 2 summarizes the distribution by class observed in 400 pachytene spermatocytes. We found no significant differences compared to results reported for spermatocytes of different animals including those treated with CBG banding (not shown). We found that $87 \%$ of the spermatocytes were in Classes 3-7, with Classes 4 and 5 being the most common $(47 \%)$. We did not find any spermatocytes in Classes 1 or 12-19. The bar graph in Figure 5 shows the expected frequency distribution by class according to the probabilistic model as compared to the observed frequency in the spermatocytes. The results show that the actual and expected distributions are similar, with the exception that higher than expected frequencies were found for the lower classes (Fig. 5).
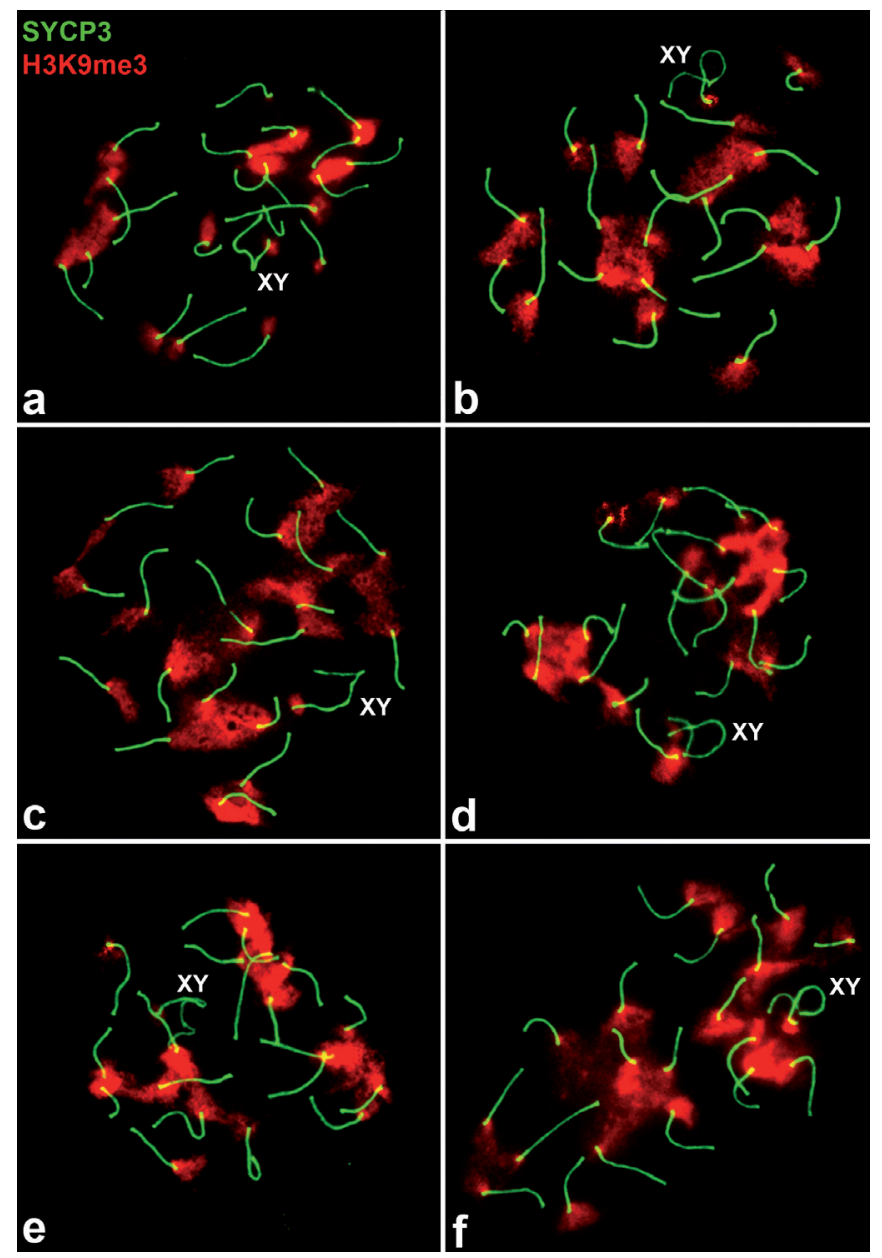

Figure 4: Combinations of single and associated CTC in spermatocyte nuclear spreads

Spermatocyte spreads of Mus domesticus $2 \mathrm{n}=40$ : The chromosomal axes were labeled with FITC anti-SYCP3 antibodies (green) and the heterochromatin of the CTC with Texas Red anti-H3K9me3 antibodies (red). For each spermatocyte, the $\mathrm{XY}$ bivalent is indicated. The class and combination of CTC associations for each spermatocyte is indicated. a) Class 5, combination: 5-4-2-2-2-1-1-1-1; b) Class 4, combination: 4-3-3-2-1-1-1-1-1-1; c) Class 3, combination: 3-3-2-2-2-2-1-1-1-1-1; d) Class 10, combination: 10-5-1-1-1; e) Class 6, combination: 6-5-4-1-1; f) Class 4, combination: 43-3-2-1-1-1-1-1-1-1.

Nuclear localization and associations of CTCs through meiotic prophase

In 120 3D nuclei, obtained by superimposing the different planes and various states of the meiotic prophase nucleus, we studied the trajectory of the single chromosomal axes or the $\mathrm{SC}$ and the location of the centromeres. To perform this analysis, we treated the cells in such a way to conserve the sphericity of the nuclei and used indirect immunofluorescence to simultaneously mark the centromeric regions and the axial elements of the bivalents' SC. We observed approximately 20 centromeric signals per nucleus, located in the end of each chromosome and distributed principally in the periphery of the nucleus. According to the stage of meiotic prophase, the chromosomal axes varied in length and thickness depending on whether they were single axes, (leptotene; Fig 6a and $a^{\prime}$ ), had completed synapsis (pachytene; Fig. 6b and b'), or had begun desynapsis (diplotene; Fig. $6 c$ and $c^{\prime}$ ). In early prophase nuclei, we observed single chromosomal axes formed or partially formed and detected some accumulations of the protein SYCP3. The centromeric signals aggregated in one or two main groups towards the nuclear periphery. This aggregation of centromeres may represent the chromosomal configuration known as the bouquet, in which the telomeric regions of the chromosomes all cluster over a small area of the nuclear envelope (Fig. 6a). In Mus, this is because the centromeres are located near the proximal telomere in all the bivalents. When treated with DAPI, this same nucleus showed a large DAPI-positive chromocenter, whose location coincided with the regions of aggregated centromeres (Fig. 6a'). DAPI staining reveals the DNA rich in AT sequences, which in Mus is the DNA that underlies the pericentromeric heterochromatin.

\section{Table 2}

Observed frequency of spermatocytes per class

Spermatocyte classes

1 2

3

4

5

6

7

8

9

10

11

12

13

14

15

16

17

18

19

Total 19
Observed frequency of spermatocytes per class

0
0.50
11.25
23.25
23.50
18.50
10.25
6.50
4.25
1.25
0.75
0
0
0
0
0
0
0
0

100.00 


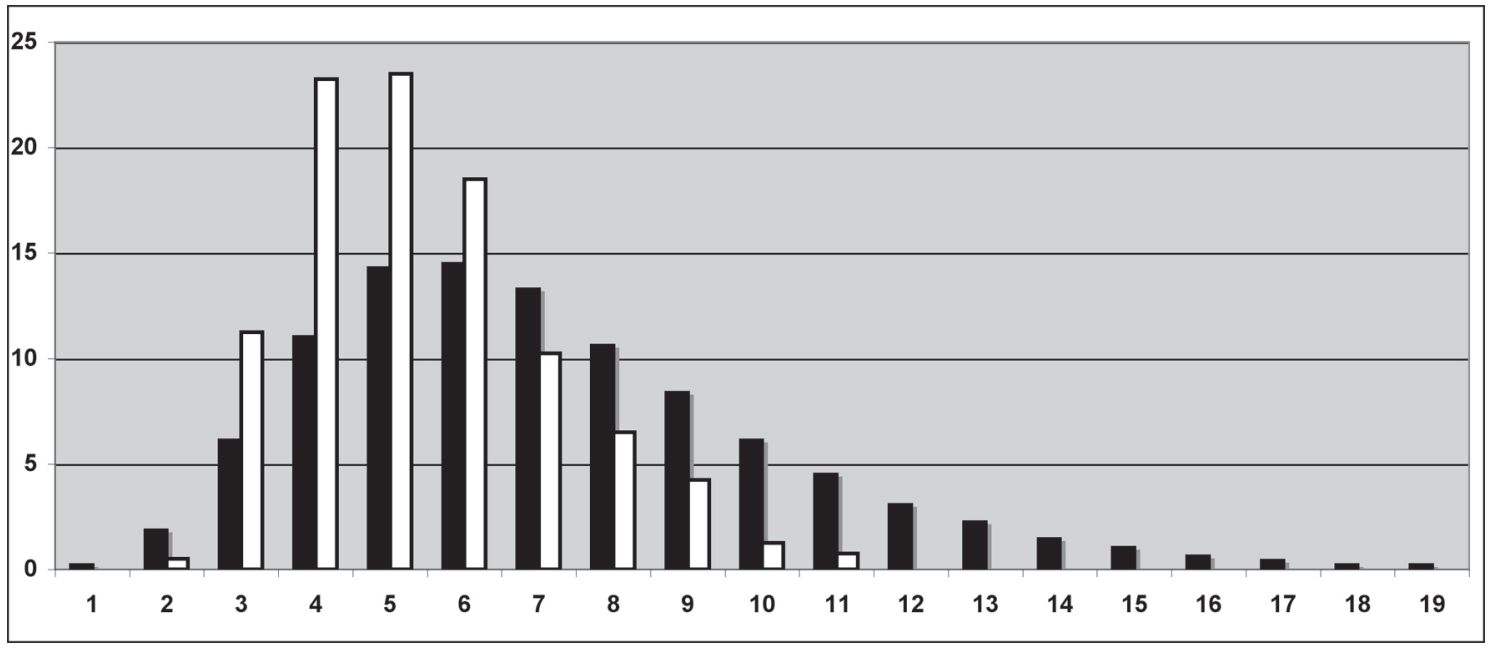

Figure 5: Comparison between expected and observed frequency (\%) of spermatocytes in 19 classes.

Graph comparing the expected frequency distribution of the 19 classes according to the probabilistic model (black bars) and the observed distribution of spermatocytes by class (white bars). To obtain the latter, we studied 400 pachytene spermatocyte spreads treated with immunocytochemical to identify the chromosomal axes and heterochromatin of each CTC. Each spermatocyte was assigned to a class according to the largest number of associated CTC it contained (Materials and Methods). The observed distribution of spermatocytes is similar to the expected distribution by chance, although the spermatocytes observed are concentrated mainly in classes with lower numbers of associated CTC.
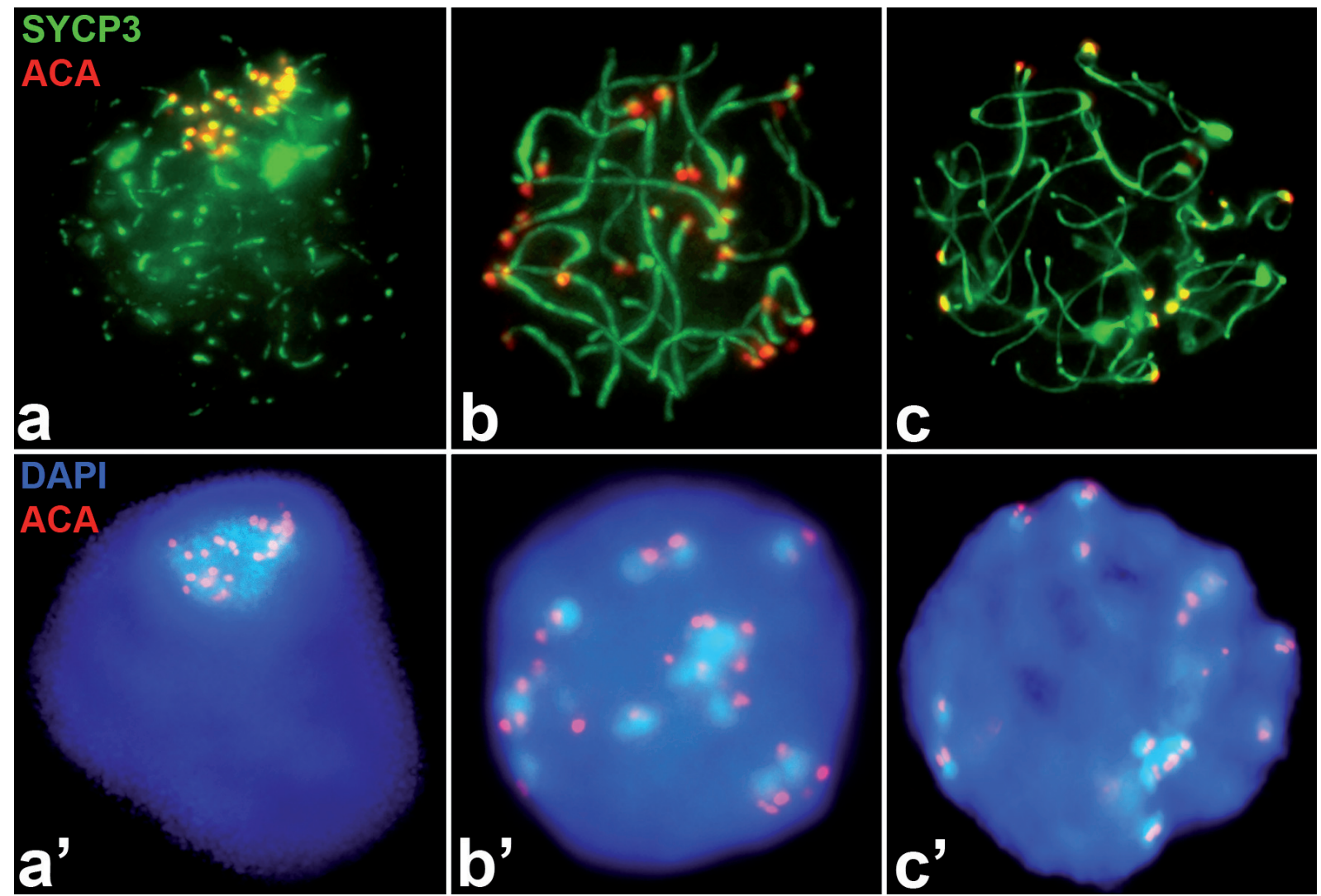

Figure 6: Nuclear localization and associations of CTC through meiotic prophase.

Total image of selected spermatocyte nuclei in a) leptotene, b) pachytene and c) diplotene:

a), b), c) The chromosomal axes were labeled with FITC anti SYCP3 antibodies (green) and the centromeres with Texas red anti centromere antibodies (ACA) (red); d), e), f) the centromeres appear labeled in red and DNA counterstained with DAPI (blue). a) chromosomal axes are in formation and the centromeric signals appear aggregated at the nuclear periphery; $\left.a^{\prime}\right)$ centromeres and heterochromatin (DAPI+) can be seen together forming one huge chromocenter; b) chromosomal axes are in synapsis and the centromeres, single or associated, are at the nuclear periphery; $b^{\prime}$ ) centromeres and heterochromatin are single or associated, forming many chromocenters; c) chromosomal axes are in partial desynapsis, the distal and centromeric regions still in synapsis; $c^{\prime}$ ) most of the centromeres/heterochromatin are single, although some of them appear associated. 
In pachytene nuclei, we found that the SC of the bivalents was distributed throughout the nuclear space. The centromeric signals were observed in one end of each bivalent in the nuclear periphery, whether single or aggregated in groups of two or more. The most common combinations of associations observed in the spermatocytes were groups of 4 or 5 centromeres. The DAPI chromocenters appeared in larger numbers and smaller sizes than in earlier stages of prophase, and their location within the nuclei coincided with that of the aggregated centromeric regions or associated CTC (Fig. $6 \mathrm{~b}$ and $\mathrm{b}^{\prime}$ ).

In diplotene nuclei, we observed short terminal segments of SC and extensive lateral elements in desynapsis in most bivalents. The fluorescent signals that identify the centromeric regions were mainly toward the nuclear periphery and less aggregated than previously. The DAPI reaction revealed numerous small chromocenters that coincided with the locations of single or associated centromeric regions (Fig. $6 \mathrm{c}$ and $\mathrm{c}^{\prime}$ ).

In cells undergoing metaphase I of meiosis, treated with CBG banding, the intense dark reaction of the pericentromeric heterochromatin in the ends of the dividing bivalents was visible. In the numerous metaphases observed, one or more bivalents were often linked to bridges of positive heterochromatin CBG banding (Fig. 7).

\section{DISCUSSION}

The proximal ends of all Mus domesticus $2 n=40$ autosomal bivalents are similar in size and structure, made up of a proximal telomere, centromere, and abundant pericentromeric heterochromatin, an arrangement which we call the Centromere Telomere Complex (CTC). In these nuclei, the bivalents bind to the nuclear envelope via their

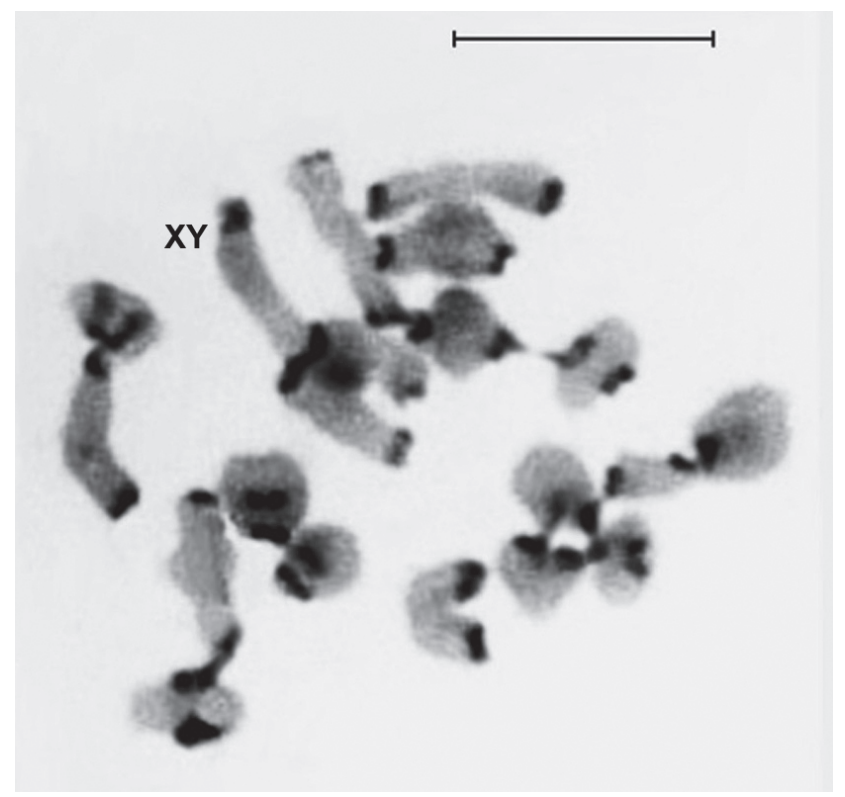

Figure 7: CBG banding on metaphase I chromosomes of Mus domesticus $\mathbf{2 n}=\mathbf{4 0}$ spermatocytes

Shows the ectopic unions among one or more chromosomes mediated by the CBG-positive pericentromeric heterochromatin. $\mathrm{XY}$, sex chromosomes. telomeric ends during meiotic prophase. Therefore, in $M$. domesticus spermatocytes, the CTC of all the bivalents are located on the nuclear periphery (Berrios et al., 1999; 2004). It is in this space, defined mainly by the limit imposed by the nuclear envelope, that we observed the unions or associations among the CTC of the autosomal bivalents. Although a large portion of the XY bivalent's chromatin also occupies the nuclear periphery, we did not observe frequent associations between the CTC and the sexual bivalent. Chromosomal territories that occupy the same nuclear space have the opportunity to establish contact with each other. These contacts can become more permanent associations if their structural and functional properties favor the maintenance of these unions. This is the case for human nucleolar bivalents, which associate in groups of 2, 3, 4, or 5 in the nuclear periphery, organizing a common nucleolus secured by the heterochromatin of the short arms of said bivalents (Stahl et al., 1984; Berríos and Fernández-Donoso 1990).

Origin of associations among CTC

It has been proposed that these associations emerge because the separate associable elements can approach and thereby come into contact with one another (Stahl et al., 1984). It has been reported that during pachytene, SC exhibited dramatic and continuous movement throughout the nucleus, traversing relatively large distances while twisting, folding, and unfolding (Scherthan et al., 2007). Chromosomal displacement and movements may contribute to the contact among chromosomal territories; however, in this study we propose these movements, in combination with the increased size in nuclear volume, contribute to disaggregation of the bivalents. Nevertheless, it is possible that new aggregations form during pachytene.

At the leptotene/zygotene transition of the meiotic prophase, telomeres form a polarized organization, called the bouquet. All chromosome ends attach to the inner surface of the nuclear envelope and aggregate within a limited area. The bouquet stage is essentially contemporaneous with the onset of pairing, synapsis and SC formation (Zickler and Kleckner, 1998; Harper et al., 2004). According to our observations, the associations originate during the configuration of the bouquet that chromosomes adopt during early prophase. During this close meeting of chromosomes, the physical proximity of their proximal ends may result in the transient formation of a large heterochromatin chromocenter situated in the nuclear periphery, determined by the telomeres' attachment to the nuclear envelope and the terminal position of the CTC in the bivalents. These conditions favor the aggregation of heterochromatin and may give rise to a random distribution of the various bivalents. In this way, the chromocenters present in the pachytene nuclei are the result of the random disaggregation of the chromocenter or cluster formed during the bouquet. A comparable situation has been proposed for Mus domesticus somatic nuclei, in which the heterochromatin chromocenters originate when the block of heterochromatin produced during Rabl configuration during mitotic anaphase fragments (Cerda et al, 1999). In spite of differences between the situations, in both cells the nuclear architecture originates in the Rabl configuration of the somatic nuclei and 
the configuration of the bouquet during meiosis. Obviously, this nuclear organization is more prominent in the heterochromatin-rich Mus chromosome/bivalent; however, it is possible that a similar organization is present in the nuclei of other species, with major and minor variations determined by the characteristics of their chromosomes (Berríos et al., 1999; 2004).

\section{Quantification of associations of CTC in the spermatocyte}

The quantitative results show that the CTC of the 19 Mus bivalents form associations within each spermatocyte in a roughly similar fashion to the potential combinations of 19 equivalent elements dispersed randomly over a limited area of the nuclear envelope. No significant differences in the distribution of spermatocytes per Class between individuals were observed. Although the observed frequency distribution for classes of CTC associations was similar to that predicted in the probabilistic model, there were fewer small associations of CTC than predicted. It is important to keep in mind that the disaggregation of CTC is progressive and concomitant with the development of prophase. Therefore, the associations observed correspond more fully with those predicted by the probabilistic model than if the quantification had been performed before pachytene.

It is also possible that the method used to quantify the observed associations contributed to the differences. In effect, although we attempted to preserve the associations, the unavoidable artifact of fixation, along with the destruction of the nuclear envelope, may have resulted in a loss of associations among higher numbers of CTC. In spite of the limitations discussed, the comparative analysis of the distribution of associations suggests that these may have been produced by chance within the spermatocyte.

Furthermore, our observations also show that some of the associations between bivalents present in prophase persist until metaphase I. These unions were maintained despite the technical disruption created by preparing the spreads, suggesting that the physical union among the chromosomes is strong. The quantitative analysis performed on the bivalents in prophase quantified how many elements (CTC) formed associations, not which elements were associated. We cannot discount the possibility that there were preferential associations among some bivalents or chromosomes; that is, that the CTC were in fact distinguishable for the effect of the associations. In this way, variability in CTC associations among spermatocytes from the same or different individuals could become present. There is some data to suggest that there are specific associations among nucleolar bivalents, between nucleolar bivalents and the XY bivalent (Berríos et al., 1990; Tres, 2005), and between bivalent 15 and the XY bivalent (Metzler-Guillemain et al., 1999). If there were preferential associations among some chromosomes in the Mus spermatocytes, and these persisted through metaphase $\mathrm{I}$, these associations may influence the distribution of these chromosomes in the meiotic spindle and their eventual normal segregation.

Consequences of associations among CTC

It has been shown that in mouse telocentric chromosomes, telomere/centromere sequences exhibit the same polarity and a high sequence identity of $>99 \%$ between nonhomologous chromosomes. It has been proposed that this organization reflects a mechanism of frequent recombinational exchange among non-homologous chromosomes that should promote the stable evolutionary maintenance of a telocentric karyotype (Kalitsis et al., 2006). The sequence homogenization of telocentric chromosomes, or sequence coincident evolution of telocentric karyotypes might have occurred by means of multiple small exchanges among these large tracts of tandemly repeated DNA. The associations among the CTC of the autosomal bivalents during meiotic prophase here described would provide the necessary physical proximity for these frequent recombinational exchanges between non-homologous chromosomes to occur. Subsequently, these recombined chromosomes would be passed on to the offspring through the resulting gametes, thus representing a mechanism for the sequence homogenization that would occur throughout the cell generations.

A similar origin has been proposed for the concerted evolution of the ribosomal DNA spacers on non-homologous nucleolar chromosomes (Arnheim et al., 1980; Gonzalez, and Sylvester., 2001; Eickbush and Eickbush, 2007). Such rDNA homogenization has been principally explained by recombination among nucleolus organizers of nonhomologous chromosomes (Gonzalez, and Sylvester, 2001), and it would be related to the well-known phenomenon of acrocentric chromosomes that associate to form a common nucleolus. In this chromosomal arrangement, the physical proximity of rDNA from different nucleolar organizer regions can give rise to genetic exchanges between non-homologous nucleolar chromosomes (Berrios and Fernandez-Donoso, 1990). Furthermore, it has been described that the rDNA in various strains of $M u s$ is found not only in NOR regions of established nucleolar chromosomes, but also in some copies of the heterochromatin of other non-nucleolar chromosomes (Rowe et al., 1996; Ito el al., 1996). Given the organization of the Mus nucleolar bivalents, in which NOR regions localize in the proximal end of the chromosomes contiguous with the heterochromatin, it is possible that frequent and random associations of CTC, in which the nucleolar bivalents also participate, are the most favorable chromosomal configuration for rDNA to propagate toward the heterochromatin of non-nucleolar chromosomes.

The chromosomal exchange may be either quite small, like the referred recombination between non-homologous CTC, or quite extensive, like the whole chromosomal arms that occur in Mus Robertsonian translocations (Garagna et al., 2001; Garagna et al., 2002). Multiple associated bivalents as the chromosomal configuration pattern also provide the nuclear scenario in which rearrangements among whole chromosomal arms could arise. In fact, the minor and major satellite DNA, which directly participate in such chromosomal reorganization, form part of the heterochromatin cluster of associated CTC.

From this point of view, it is not necessary to suggest an antagonistic mechanism for both the stable maintenance of the Mus telocentric karyotype and the occurrence of whole-arm Robertsonian translocations (Kalitsis et al., 2006). The nuclear architecture of the Mus spermatocytes where associations between bivalents are 
the normal pattern could provide an appropriate environment where both types of chromosomal exchanges may arise (Redi et al., 2001).

The model is very useful for studying the nuclear architecture, as it provides a proposed structure for the participating units (CTC), as well as a concept of nuclear spaces as areas with varying probabilities of being occupied and hosting associations among chromosomal territories, according to the temporal progress of the prophase. It also allows for visualization of a meiotic nuclear architecture that is not encoded genetically, but is based on higher-order patterns of spatial associations among specific chromosome domains.

\section{ACKNOWLEDGEMENTS}

This work was supported by FONDECYT Project \# 1080090 and Grant A/017762/08 from Agencia Española de Cooperación Internacional para el Desarrollo (Spain).

\section{REFERENCES}

ARNHEIM N, KRYSTAL M, SCHMICKEL R, WILSON G, RYDER O, ZIMMER E (1980) Molecular evidence for genetic exchanges among ribosomal genes on nonhomologous chromosomes in man and apes. Proc Natl Acad Sci U S A. 77(12): 7323-7.

ALCOBIA I, DILAO R, PARREIRA L (2000) Spatial associations of centromeres in the nuclei of hematopoietic cells: evidence for celltype-specific organizational patterns. Blood 95(5): 1608-1615.

BEIL M, FLEISCHER F, PASCHKE S, SCHMIDT V (2005) Statiscal analysis of the three-dimensional structure of centromeric heterochromatin in interphase nuclei. Journal of microscopy $217(1)$ 60-68.

BERRIOS S, FERNÁNDEZ-DONOSO R (1990) Nuclear architecture of human pachytene spermatocytes. Hum Genet 86: 103-116.

BERRIOS S, FERNÁNDEZ-DONOSO R, AYARZA E, PAULOS A, MORENO M (1999) Non-random distribution of the pericentromeric heterochromatin in meiotic prophase nuclei of mammalian spermatocytes. Genetica 106: $187-195$

BERRÍOS S, FERNÁNDEZ-DONOSO R, PINCHEIRA J, PAGE J, MANTEROLA M, CERDA MC (2004) Number and nuclear localisation of nucleoli in mammalian spermatocytes. Genetica 121(3): 219-28.

BRINKLEY BR, BRENNER SL, HALL JM, TOUSSON A, BALCZON RD, VALDIVIA MM (1986) Arrangements of kinetochores in mouse cells during meiosis and spermiogenesis. Chromosoma 94(4): 309-317.

CERDA MC, BERRIOS S, FERNÁNDEZ-DONOSO R, GARAGNA S, REDI C (1999) Organization of complex nuclear domains in somatic mouse cells. Biol Cell 91: 55 - 65 .

CREMER T, KUPPER K, DIETZEL S, FAKAN S (2004) Higher order chromatin architecture in the cell nucleus: on the way from structure to function. Biol Cell 96(8): 555-67.

CREMER T, CREMER M, DIETZEL S, MULLER S, SOLOVEI I, FAKAN S (2006) Chromosome territories - a functional nuclear landscape. Curr Opin Cell Biol 18(3): 307-316.

DERNBURG AF, BROMAN KW, FUNG JC, MARSHALL WF, PHILIPS J, AGARD DA, SEDAT JW (1996) Perturbation of nuclear architecture by long distance chromosome interactions. Cell 85: 745-759.

DE WIT E, VAN STEENSEL B (2009) Chromatin domains in higher eukaryotes: insights from genome-wide mapping studies. Epub 118(1): 25-36.

EICKBUSH TH, EICKBUSH DG (2007) Finely orchestrated movements: evolution of the ribosomal RNA genes. Genetics 175(2): 477-85.

FERNÁNDEZ-DONOSO R (1982) Asociaciones cromosómicas en el núcleo de los meiocitos y reordenamientos cromosómicos. Actas $\mathrm{V}$ Congr Latinoam Genet pp 105-114.

GARAGNA S, MARZILIANO N, ZUCCOTTI M, SEARLE JB, CAPANNA E, REDI CA (2001) Pericentromeric organization at the fusion point of mouse Robertsonian translocation chromosomes. Proc Natl Acad Sci USA 98(1): 171-175
GARAGNA S, ZUCCOTTI M, CAPANNA E, REDI CA (2002) Highresolution organization of mouse telomeric and pericentromeric DNA. Cytogenet Genome Res 96(1-4): 125-9.

GÖNDOR A, OHLSSON R (2009) Chromosome crosstalk in three dimensions. Nature 46: 212-217.

GONZALEZ IL, SYLVESTER JE (2001) Human rDNA: evolutionary patterns within the genes and tandem arrays derived from multiple chromosomes. Genomics. 73(3): 255-63.

GUENATRI M, BAILLY D MAISON C, ALMOUZNI G (2004) Mouse centric and pericentric satellite repeats form distinct functional heterochromatin. J Cell Biol 166: 493-505.

HAAF T, SCHMID M (1991) Chromosome topology in mammalian interphase nuclei. Exp Cell Res 92: 325-332.

HARPER L, GOLUBOVSKAYA I, CANDE WZ (2004) A bouquet of chromosomes. J Cell Sci 117: 4025-32.

HEYTING C (1996) Synaptonemal complexes: structure and function. Curr Opin Cell Biol 8: 389-396.

ITO T, TSUCHIYA K, OSAWA S, SHIBATA H, KANDA N (2008) Mapping of rRNA gene loci in the mice, Mus musculus molossinus (Japan) and Mus musculus musculus (Russia) by double color FISH. J Vet Med Sci 70(9): 997-1000.

JOSEPH A, MITCHELL AR, MILLER OJ (1989) The organization of the mouse satellite DNA at centromeres. Exp Cell Res 183: 494-500.

KALITSIS P, GRIFFITHS B, CHOO KH (2006) Mouse telocentric sequences reveal a high rate of homogenization and possible role in Robertsonian translocation. Proc Natl Acad Sci USA 103(23): 87868791.

LAMMERS JHM, OFFENBERG HH, VAN AALDEREN M, VINK ACG, DIETRICH AJJ, HEYTING C (1994) The gene encoding a major component of the lateral elements of synaptonemal complexes of the rat is related to X-linked lymphocyte-regulated genes. Mol Cell Biol 14: $1137-1146$

LÓPEZ-FENNER JE, FERNÁNDEZ-DONOSO R (1991) Modelos probabilísticos para la asociación al azar de los complejos centrómero-teloméricos en el paquiteno del espermatocito de Mus domesticus. Revista Frontera, Temuco (Chile) 9-10: 43-51.

MANUELIDIS L (1985) Individual interphase chromosomes revealed by in situ hybridization. Hum Genet 71: 288-293.

MANUELIDIS L, BORDEN J (1988) Reproducible compartmentalization of individual chromosome domains in human CNS cells revealed by in situ hybridization and three dimensional reconstruction. Chromosoma 96: 397-410.

MANUELIDIS L (1990) A view of interphase chromosomes. Science 250: 1533-1540.

MAYER R, BRERO A, VON HASE J, SCHROEDER T, CREMER T, DIETZEL $S$ (2005) Common themes and cell type specific variations of higher order chromatin arrangements in the mouse. BMC Cell Biol. 7: 6: 44 .

MAYFIELD JE, ELLISON JR (1975) The organization of interphase chromatin in Drosophilidae. Chromosoma 52: 37-48.

METZLER-GUILLEMAIN C, MIGNON C, DEPETRIS D, GUICHAOUA MR, MATTEI MG (1999) Bivalent 15 regularly associates with the sex vesicle in normal male meiosis. Chromosome Res 7(5): 369-378.

MISTELI T (2008) Cell biology: nuclear order out of chaos. Nature 456(7220): 333-334.

NAGELE R, FREEMAN T, MCMORROW L, LEE HY (1995) Precise spatial positioning of chromosomes during prometaphase: evidence for chromosomal order. Science 270: 1831-1835.

OFFENBERG HH, SCHALK JA, MEUWISSEN RL, VAN AALDEREN M, KESTER HA, DIETRICH AJ, HEYTING C (1998). SCP2: a major protein component of the axial elements of synaptonemal complexes of the rat. Nucleic Acids Res 26: 2572-2579.

PAGE J, SUJA JA, SANTOS JL, RUFAS JS (1998) Squash procedure for protein immunolocalization in meiotic cells. Chromosome Research 6: 639-642.

PARDUE ML, GALL JG (1970) Chromosomal localization of mouse satellite DNA. Science 170: 1356-1358.

PETERS AH, PLUG AW, VAN VUGT MJ, DE BOER P (1997) A dryingdown technique for the spreading of mammalian meiocytes from the male and female germline. Chromosome Res 5: 66-68.

REDI CA GARAGNA S, ZACHARIAS H, ZUCCOTTI M, CAPANNA E (2001) The other chromatin. Chromosoma 110: 136-147.

ROWE LB, JANASWAMI PM, BARTER ME, BIRKENMEIER EH (1996) Genetic mapping of $18 \mathrm{~S}$ ribosomal RNA-related loci to mouse chromosomes 5, 6, 9, 12, 17, 18, 19, and X. Mamm Genome. 7(12): 886889. 
SADONI N, LANGER S, FAUTH C, BERNARDI G, CREMER T, TURNER BM, ZINK D (1999) Nuclear organization of mammalian genomes: Polar chromosome territories build up functionally distinct higher order compartments. J Cell Biol 146: 1211-1226.

SCHERTHAN H (2001) A bouquet makes ends meet. Nat Rev Mol Cell Biol 2: 621-627.

SCHERTAN H, WANG H, ADELFALK C, WHITE EJ, COWAN C, CANDE WZ, KABACK DB (2007) Chromosome mobility during meiotic prophase in Saccharomyces cerevisiae. Proc Natl Acad Sci USA 104(43): 16934-16939.

SOLARI AJ, TRES L (1970). The three dimensional reconstruction of the XY chromosomal pair in human spermatocytes. J Cell Biol 45: 43-53.

SOLARI AJ (1982) Recombination bars in human synaptonemal complexes spread with sodium dodecyl sulphate. Actas V Congr Latinoam Genet pp. 115-123.

STAHL A, HARTUNG M, DEVICTOR M, BERGE-LEFRANC JL (1984) The association of the nucleolus and the short arm of acrocentric chromosomes with the XY pair in human spermatocytes: its possible role in facilitating sex-chromosome acrocentric translocations. Hum Genet 68: 173-180.

TAKIZAWA T, MEABURN KJ, MISTELI T (2008) The meaning of gene positioning. Cell 135(1): 9-13.

TRES LL (2005) XY chromosomal bivalent: nucleolar attraction. Mol Reprod Dev. 72(1): 1-6.

YASMINEH WG, YUNIS JJ (1970) Localization of mouse satellite DNA in constitutive heterochromatin. Exp Cell Res 59(1): 69-75.

VON WETTSTEIN D, RASMUSSEN SW, HOLM PB (1984) The synaptonemal complex in genetic segregation. Annu Rev Genet 18: 331-413.

YANG F, WANG PJ (2009) The Mammalian synaptonemal complex: a scaffold and beyond. Genome Dyn 5: 69-80.

ZICKLER D, KLECKNER N (1998) The leptotene-zygotene transition of meiosis. Annu Rev Genet 32: 619-97.

ZUCCOTI M, PICCINELLI A, GIORGI RP, GARAGNA S, REDI CA (1995) Chromatin organization during mouse oocyte growth. Mol Reprod Dev 41(4): 479-485 
